\title{
Trunk Muscle Training, Posture Fatigue, and Performance in Laparoscopic Surgery
}

\author{
MICHAEL A. TSE, M.B.A., B.Sc., ${ }^{1}$ R.S.W. MASTERS, D.Phil., ${ }^{1}$ \\ A.M. McMANUS, Ph.D., ${ }^{1}$ C.Y. LO, M.S., FRCS. ${ }^{2}$ and N.G. PATIL, M.S., FRCS (ED) ${ }^{3}$
}

\begin{abstract}
Purpose: To investigate the effect of trunk muscle endurance training on the perception of back postural fatigue and performance of a laparoscopic task.

Materials and Methods: Thirty-one medical students (18 men and 13 women) with no laparoscopic surgical experience were randomly assigned to either a training group or a control group. Participants in the training group underwent a 6-week, 18-session trunk (abdominal and back muscle) endurance training program, whereas participants in the control group did not. Performance by all participants was assessed on a simulated laparoscopic task under varying conditions of low back postural fatigue, both before and after the training program.

Results: Participants in the training group showed significant improvements in trunk endurance after the 6-week trunk endurance training program $(P<0.05)$, whereas those in the control group did not. The improvements in trunk endurance were accompanied by significantly reduced perceptions of discomfort $(P<$ $.001)$ and fewer errors during performance of the laparoscopic task $(P<0.02)$, whereas no significant changes occurred for the control group $(P>0.05)$.

Conclusion: Increasing trunk endurance can reduce postural fatigue and discomfort during simulated laparoscopic tasks, which may assist in the management of errors during laparoscopy.
\end{abstract}

\section{INTRODUCTION}

D URING THE PAST TWO DECADES, minimally invasive surgery by laparoscopy has significantly revolutionized surgical practice, becoming the "gold standard" for various surgical procedures. The physical demands of minimally invasive surgery on the surgeon can be high, such as during some laparoscopic urologic procedures. Surgeons frequently stand for extended periods and assume atypical postures with prolonged flexion of the torso and neck. ${ }^{1,2}$ Because of the muscle fatigue that accompanies these postures, pain and discomfort are often reported, ${ }^{3-12}$ and work-related injuries may occur. ${ }^{13}$ Furthermore, fatigue has an effect on manual dexterity, ${ }^{14}$ an important component of laparoscopic procedures.

The purpose of this study was to evaluate the potential effect of a 6-week trunk endurance training program on a simulated laparoscopic task. Numerous ergonomic studies have been conducted to investigate the origins of physical problems in surgeons who complain of fatigue and discomfort during laparoscopic surgery, ${ }^{4,5,10-13}$ and even to investigate ways to reduce postural fatigue by using ergonomic supports. ${ }^{15}$ To our knowledge, this is the first study that attempts to manipulate the physical training status of a person's trunk muscles to address the issue of postural fatigue during laparoscopic performance.

\section{MATERIALS AND METHODS}

\section{Participants}

Thirty-one healthy preclinical medical students (18 men and 13 women) with no previous surgical experience were invited to participate in this study on a voluntary basis. Right-hand dominance was self-reported by all participants. Ages ranged

${ }^{1}$ Institute of Human Performance, ${ }^{2}$ Jockey Club Skills Development Centre, Department of Surgery, and ${ }^{3}$ Centre for Education and Training, Department of Surgery, The University of Hong Kong, Hong Kong, China. 
from 20 to 32 years (mean 22.1 years; standard deviation 2.8 years).

Participants were randomly assigned to a training group (TG), consisting of nine men and seven women, who took part in a specialized trunk training intervention, or to a control group (CG), consisting of nine men and six women who did not receive training. No significant differences existed between the groups for height, body weight, body mass index, or physical activity levels $(P>0.05)$.

Participants were informed of the experimental procedures and the potential risks via written and verbal explanations. Each participant gave written consent before participation in the study. Participants were screened for low-back pain, activity levels, and musculoskeletal or cardiovascular disorders by means of physical activity readiness and health questionnaires. Scoliosis, back pain, or spinal surgeries were used as exclusion criteria. The study was approved by the Institutional Review Board.

\section{General study design}

An initial clinical assessment to measure trunk endurance was performed on all participants. After these tests, 3 to 5 days of rest was allowed before participants performed the simulated laparoscopic task. Participants were asked to give feedback about their perceptions of fatigue by means of the Borg-CR 10 perceived exertion scale. ${ }^{16}$ After completing the laparoscopic task under nonfatigued conditions, participants underwent a maximal back extension fatigue protocol, ${ }^{17}$ and then immediately repeated the laparoscopic task.

A 6-week (3 days per week) training program was then undertaken by TG participants to improve muscular endurance of the trunk, i.e., low-back extensors and abdominis muscles. CG participants did not perform any specific training regimen for their trunk muscles but were allowed to continue normal recreation activities during the 6-week period. After conclusion of the 6-week period, all tests were repeated by all participants. The overall study design is shown in Fig. 1.

\section{Trunk endurance tests}

Trunk muscle endurance was assessed using four clinically reliable trunk endurance tests: back extensor, abdominal fatigue, and the right- and left-side bridge tests. ${ }^{18-20}$

\section{Apparatus and task description}

The laparoscopic task was performed on a bench model laparoscopic training box, using a 10-mm 0-degree laparoscope (Karl Storz Endoscopy, Germany) with light source (Quantum 4000, Stryker Endoscopy, Germany). A medical video camera (Stryker 814) transmitted the image via optical interface to a 58-cm laparoscopic television monitor (Sony HR Trinitron, Sony Corp, Japan) during the laparoscopic task.

Participants assumed a standard position, with the upper torso flexed at the hips by 25 degrees and were required to transfer small beads with two laparoscopic graspers from one position to another $(16 \mathrm{~cm})$ through a hoop $(5 \mathrm{~cm}$ diameter $)$. Participants were only able to view the task occurring inside the training box via a video monitor placed just below eye level at
$150 \mathrm{~cm}$ in front of the participant. A slightly flexed, standard position was adopted to consistently maintain back-muscle fatigue and prohibit participants from attaining intermittent relief by shifting positions, thus better controlling fatigue accumulation.

Participants completed 10 bead transfer practice trials of a familiarization task at their own pace, and another two sets of 20 practice trials in which they were asked to complete the task as quickly and as accurately as possible. After familiarization, participants carried out 20 trials for the actual test. A back extension fatigue protocol was administered immediately thereafter, followed by a further 20 test trials. Participants verbally estimated their perceptions of fatigue on the modified Borg-CR 10 pain/discomfort scale, using values from 0 to 10 , signifying from "nothing at all" to "extremely strong - near maximal fatigue." 16

Performance was assessed by measuring the total time taken to transfer each set of 20 beads and the total number of errors committed (eg, failed attempts to grasp a bead, dropped beads, contacts with the hoop). Each session was videotaped for assessment purposes.

\section{Back extension fatigue protocol}

Participants were required to lie prone on a plinth and perform a series of 90-degree trunk extension-flexion movements at a slow, deliberate tempo (cadence: 20 repetitions per minute), as used by Gorelick and associates. ${ }^{17}$ The fatigue protocol was terminated when the participant could no longer maintain the required cadence or reported unacceptable discomfort. The protocol was identical both pretraining and post-training.

\section{Trunk training intervention}

TG participants underwent a trunk training program validated by Tse and colleagues ${ }^{21}$ that required them to train under supervision three times per week ( $45 \mathrm{~min} / \mathrm{session}$ ) for a 6 week period (18 training sessions) and focused on a variety of simple exercises that trained the trunk muscles. All training participants were exposed to the same protocol but were challenged according to their individual progress.

\section{RESULTS}

The data were examined using analysis of variance with repeated measures and $t$ tests. Significance was accepted at $P<$ 0.05 .

\section{Trunk endurance tests}

TG participants improved significantly $(P<0.01)$ on all trunk endurance tests after the 6-week training program, whereas CG participants showed no significant changes $(P>$ 0.05) during this period (Fig. 2).

\section{Perceived exertion}

Pretraining program: No differences existed in the way TG participants group and CG participants rated their discomfort when completing the laparoscopic task before the fatigue pro- 


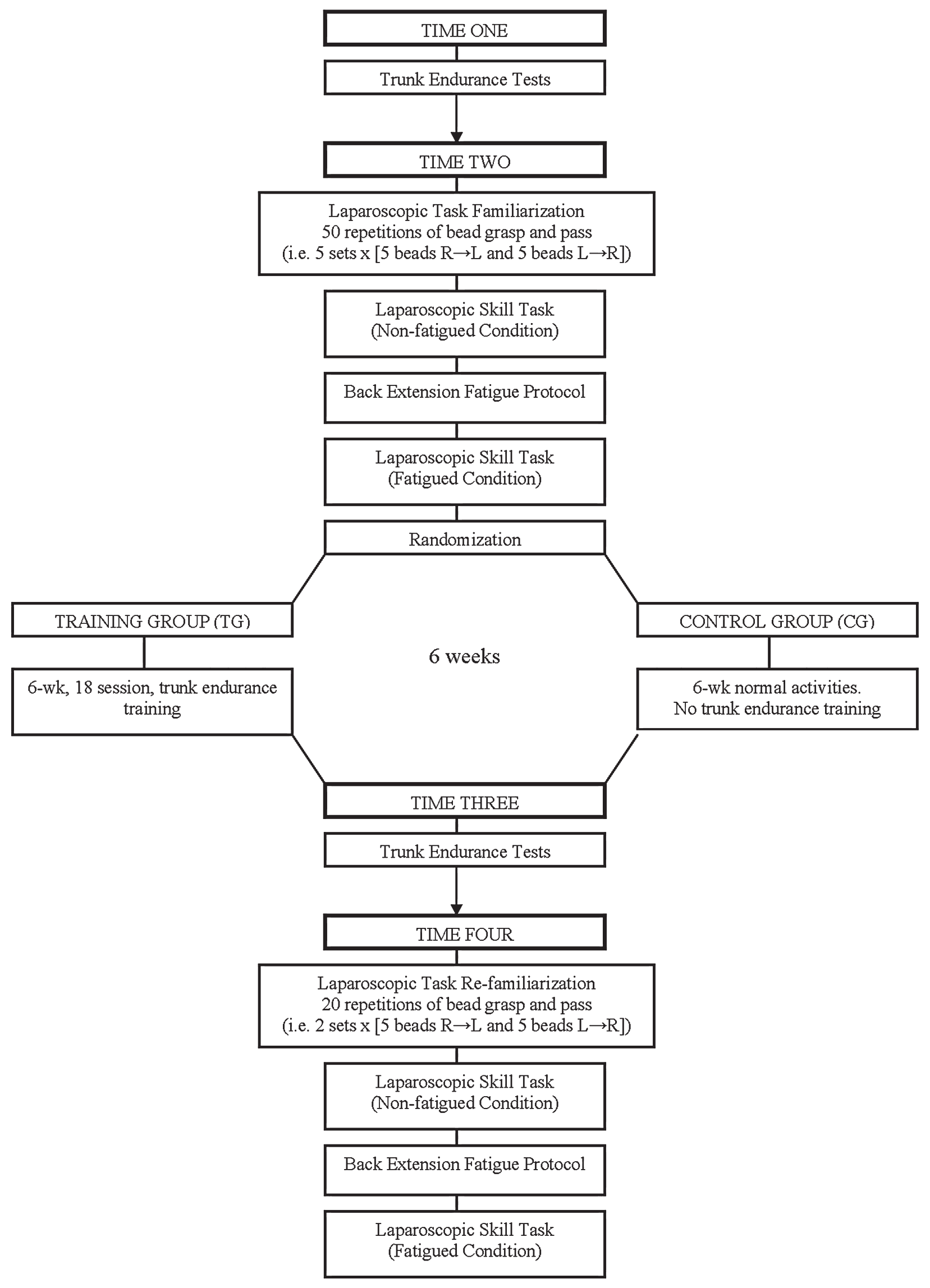

FIG. 1. Experimental design of the study. 


\section{1 - Back Extension Test}

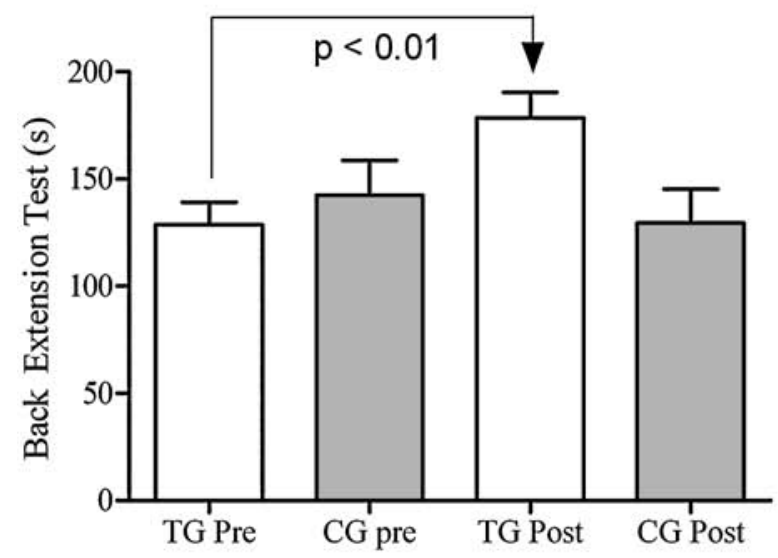

\section{3 - Right Side Bridge Test}

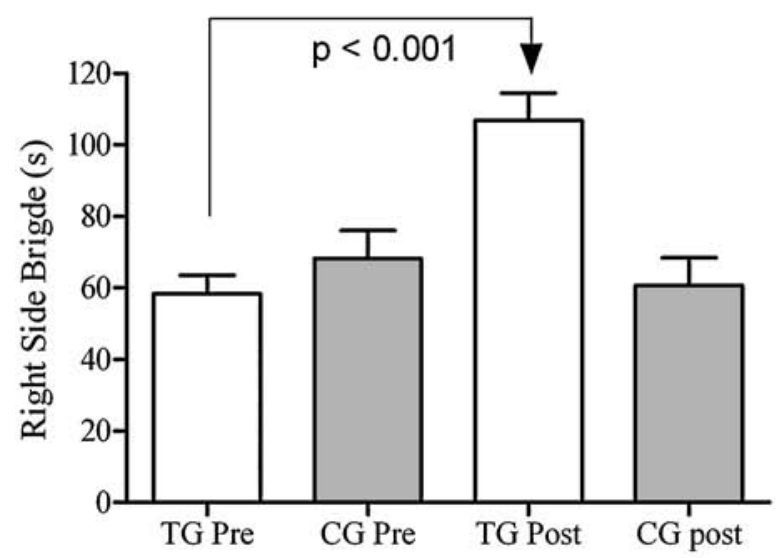

\section{2 - Abdominal Fatigue Test}

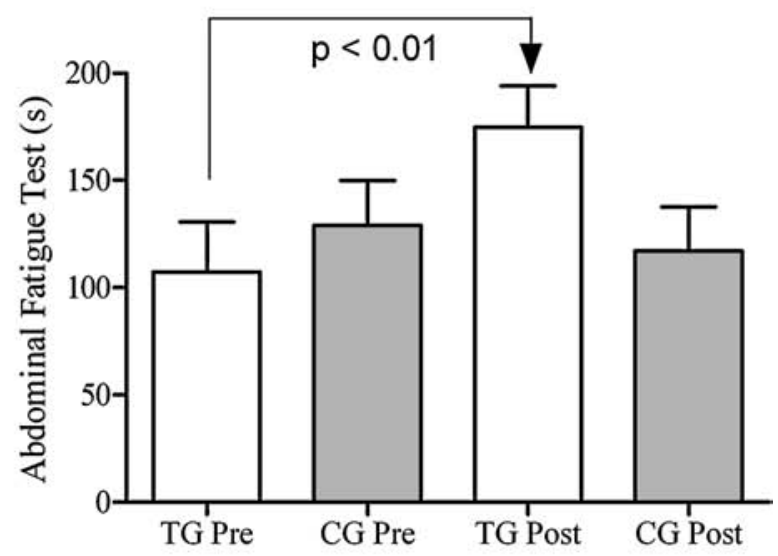

4 - Left Side Bridge Test

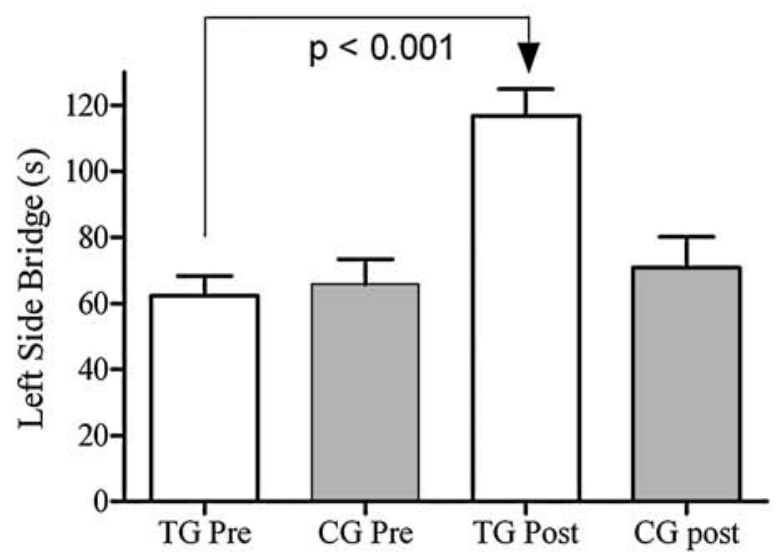

FIG. 2. Trunk endurance tests, preintervention and postintervention scores. In panels 1 to 4 , a significant improvement is apparent in the pretraining to post-training scores for each of the respective trunk tests in the $\mathrm{TG}$ only. TG $=$ training group; $\mathrm{CG}=$ control group.

tocol $(P>0.05)$. In both groups, however, a similar increase $(P<0.001)$ in discomfort ratings was evident after the fatigue protocol (Fig. 3a).

Post-training program: Changes were evident in the ratings of perceived exertion after the 6-week training program (Fig. 3b). TG participants reported significantly less discomfort from the prefatigue to the postfatigue protocol than CG participants $(P<0.03)$, which indicated that increases in discomfort were less severe in the TG.

\section{Laparoscopic task performance}

Pretraining program: Although the number of errors committed by participants in both groups decreased marginally after the fatigue protocol, no statistical differences were evident $(P>0.05)$ (Fig. 4a).
Post-training program: The TG showed a significant decrease in the number of errors committed from the prefatigue to the postfatigue protocol $(P<0.02)$, in contrast to those in the $\mathrm{CG}$, who showed no change in the number of errors $(P>$ 0.05) (Fig. 4b).

\section{Laparoscopic task completion times}

Pretraining program: The time taken to complete the task decreased significantly after the fatigue protocol in both groups $(P<0.001)$ (prefatigue: TG, 61.6 s $\pm 15.1 ; \mathrm{CG}, 62.7 \mathrm{~s} \pm 11.6$; postfatigue: TG, $50.1 \mathrm{~s} \pm 7.8$; CG, $55.9 \mathrm{~s} \pm 6.6$ ).

Post-training program: Again, the time taken to complete the task decreased significantly after the fatigue protocol in both groups $(P<0.001)$ (prefatigue: TG, 55.2 s \pm 11.6; $\mathrm{CG}, 53.9$ $\mathrm{s} \pm 8.9$; postfatigue: $\mathrm{TG}, 44.7 \mathrm{~s} \pm 10.7$; $\mathrm{CG}, 45.4 \mathrm{~s} \pm 10.7)$. 


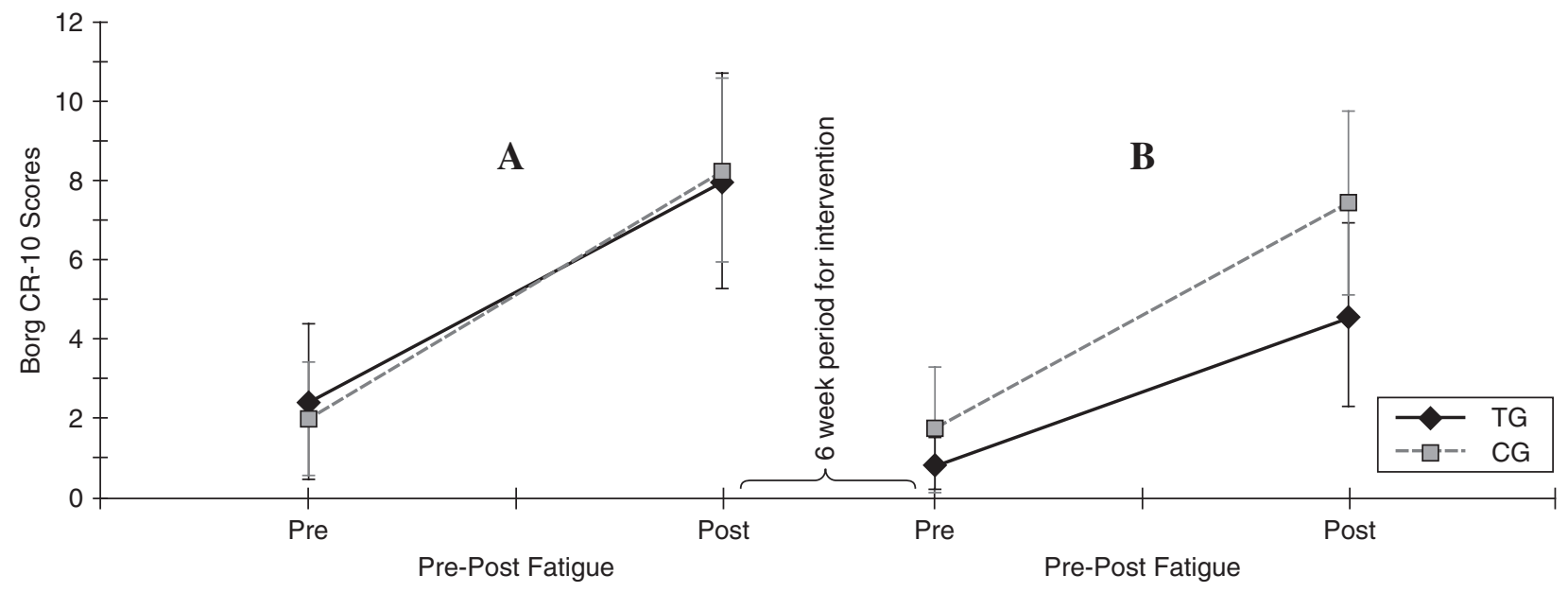

FIG. 3. Prefatigue and postfatigue-protocol Borg scores in (a) preintervention and (b) postintervention test phases. TG $=$ training group; $\mathrm{CG}=$ control group.

\section{DISCUSSION}

Musculoskeletal fatigue occurs in the back, shoulders, neck, and legs of surgeons as a consequence of prolonged flexion of the neck and torso. The primary aim of this investigation was to determine the effect of trunk muscle training on musculoskeletal fatigue and its relation to performance and discomfort during a simulated laparoscopic surgery.

Before the 6-week trunk training program, participants in both the TG and CG showed increased speed of performance when fatigued by the fatigue protocol and a marginal but nonsignificant reduction in errors. It is likely that the reduction in errors reflects continued improvements in performance that occurred regardless of fatigue.

The training program resulted in a significant improvement in trunk endurance from pretraining to post-training, accompanied by reductions in perceived discomfort when performing the laparoscopic task under fatigue conditions. Improved trunk endurance also appeared to inoculate the TG participants against increased error rates, with participants exhibiting significantly fewer errors even though they performed the task more quickly after the fatigue protocol.

CG participants also exhibited more rapid performance of the laparoscopic task after the fatigue protocol, but their error rates did not decrease. This finding may have important implications, because it suggests that persons who display high resistance to back postural muscle fatigue, as a result of trunk endurance training, possess the ability to perform a simulated laparoscopic task very rapidly without a concomitant increase in errors

It is possible that a reduction in the fatigue of trunk muscles is associated with reduced tremor of the hands, or that participants with better trunk endurance are less distracted by feelings of discomfort as they perform. Further studies investigating the

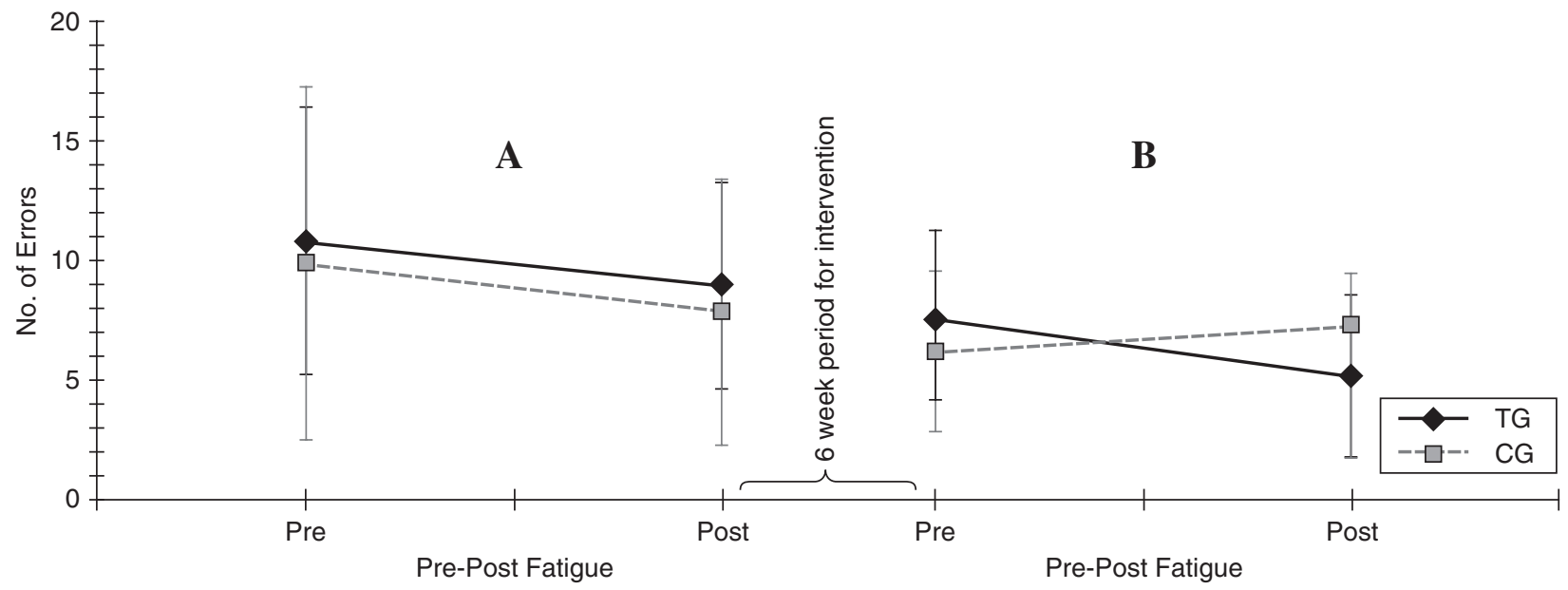

FIG. 4. Pre-error and posterror scores in (a) preintervention and (b) postintervention test phases. TG $=$ training group; CG $=$ control group. 
influence of reduced back fatigue on hand tremor and mental disposition during laparoscopic task performance are recommended. Also, to minimize potential biases in subjective ratings of discomfort, subsequent studies should blind subjects as to whether they are in a CG or TG. Future studies need also to extend our understanding of the benefits of trunk endurance training from novice medical students to experienced surgeons performing laparoscopic surgery, both in real and artificial operative settings.

\section{ACKNOWLEDGMENTS}

The authors would like to thank Dr. Hung Lau of the Tung Wah Hospital in Hong Kong and Ms. Haley Lo for their assistance, and the medical students from the University of Hong Kong, Faculty of Medicine, for their participation in the study.

\section{REFERENCES}

1. Berguer R. Surgical technology and the ergonomics of laparoscopic instruments. Surg Endosc 1998;12:458-462.

2. Cuschieri A. Whither minimal access surgery: Tribulations and expectations. Am J Surg 1995;169:9-19.

3. Berguer R, Rab GT, Abu-Ghaida H, Alarcon A, Chung J. A comparison of surgeons' posture during laparoscopic and open surgical procedures. Surg Endosc 1997;11:139-142.

4. Berguer R. Surgery and ergonomics. Arch Surg 1999;134:10111016.

5. Berguer R, Forkey DL, Smith WD. Ergonomic problems associated with laparoscopic surgery. Surg Endosc 1999;13:466-468.

6. Berguer R, Smith WD, Chung YH. Performing laparoscopic surgery is significantly more stressful for the surgeon than open surgery. Surg Endosc 2001;15:1204-1207.

7. Berguer R, Smith WD, Davis S. An ergonomic study of the optimum operating table height for laparoscopic surgery. Surg Endosc 2002;16:416-421.

8. Emam TA, Frank TG, Hanna GB, Cuschieri A. Influence of handle design on the surgeon's upper limb movements, muscle recruitment, and fatigue during endoscopic suturing. Surg Endosc 2001;15:667-672.

9. Kant IJ, de Jong LC, van Rijssen-Moll M, Borm PJ. A survey of static and dynamic work postures of operating room staff. Int Arch Occup Environ Health 1992;63:423-428.

10. Nguyen NT, Ho HS, Smith WD, Philipps C, Lewis C, De Vera RM, Berguer R. An ergonomic evaluation of surgeons' axial skele- tal and upper extremity movements during laparoscopic and open surgery. Am J Surg 2001;182:720-724.

11. Uhrich ML, Underwood RA, Standeven JW, Soper NJ, Engsberg JR. Assessment of fatigue, monitor placement, and surgical experience during simulated laparoscopic surgery. Surg Endosc 2002; 16:635-639.

12. Vereczkei A. Feussner H, Negele T, Fritzsche F, Seitz T, Bubb H, Horváth OP. Ergonomic assessment of the static stress confronted by surgeons during laparoscopic cholecystectomy. Surg Endosc 2004;18:1118-1122.

13. Udasin IG. Health care workers. Prim Care 2000;27:1079-1102.

14. Crothers IR, Gallagher AG, McClure N, James DT, McGuigan J. Experienced laparoscopic surgeons are automated to the "fulcrum effect": An ergonomic demonstration. Endoscopy 1999;31:365-369.

15. Galleano R, Carter F, Brown S, Frank T, Cuschieri A. Can armrests improve comfort and task performance in laparoscopic surgery? Ann Surg 2006;243:329-333.

16. Borg G. Borg's Perceived Exertions and Pain Scales. Champaign, IL: Human Kinetics, 1998.

17. Gorelick M, Brown JM, Groeller H. Short-duration fatigue alters neuromuscular coordination of trunk musculature: Implications for injury. Appl Ergon 2003;34:317-325.

18. McGill SM, Childs A, Liebenson C. Endurance times for low back stabilization exercises: Clinical targets for testing and training from a normal database. Arch Phys Med Rehabil 1999;80:941-944.

19. McGill SM. Low Back Disorders. Evidence-Based Prevention and Rehabilitation. Champaign, IL: Human Kinetics, 2002.

20. Biering-Sorensen F. Physical measurements as risk indicators for low-back trouble over a one-year period. Spine 1984;9:106-119.

21. Tse MA, McManus AM, Masters RS. Development and validation of a core endurance intervention program: Implications for performance in college-age rowers. J Strength Cond Res. 2005;19:547-552.

Address reprint requests to: M.A. Tse, M.B.A., B.Sc. Institute of Human Performance The University of Hong Kong 111-113 Pokfulam Road Hong Kong SAR, China

E-mail: matse@hku.hk

\section{ABBREVIATIONS USED}

$$
\begin{aligned}
& \mathrm{CG}=\text { control group } \\
& \mathrm{TG}=\text { training group }
\end{aligned}
$$

